\title{
Post-operative Transient Hypoparathyroidism: Incidence and Risk Factors
}

\author{
Jennifer Downs $^{1}$, Kerry Wilson ${ }^{2}$, Felix Made $^{2}$, Francois Malherbe ${ }^{1}$, Eugenio Panieri ${ }^{1}$, Lydia Cairncross ${ }^{1}$
}

1. Division of General Surgery, Groote Schuur Hospital, University of Cape Town, Cape Town, South Africa.

2. National Institute of Occupational Health, Johannesburg, South Africa

Correspondence to: Jennifer Downs, Email: jennifer.downs@alumnus.uct.ac.za

\begin{abstract}
Background: There is limited data on the incidence and risk factors for developing postoperative hypoparathyroidism (POHP) in the South African setting. Objectives: This study aims to calculate the incidence of postoperative hypoparathyroidism in a South African tertiary setting, and to compare local risk factors for POHP to international published data. Methods: All patients who underwent a total or completion thyroidectomy at an academic referral center from January 2010 to December 2015 were included. Data reviewed included post-operative parathyroid hormone (iPTH) level, demographics, type of operation and lymphadenectomy, size of thyroid glands resected, final histology, extra capsular extension of carcinomas, number of lymph nodes resected, and the number of parathyroid excised. Results: Postoperatively, $29 \%$ of patients were diagnosed with hypoparathyroidism. Overall, there was no association between POHP and age or gender. In patients with
\end{abstract}

\section{Introduction}

Postoperative hypoparathyroidism (POHP) is a wellrecognized complication of thyroid surgery. It can occur following thyroid surgery due to inadvertent devascularization of the parathyroid glands, unintentional excision of parathyroids, or if the parathyroid glands are deliberately excised as part of an oncological specimen(1). An early decrease in intact Parathyroid Hormone (iPTH) levels predicts postoperative hypocalcaemia with great specificity and sensitivity $(2,3)$, which can cause significant morbidity benign histology, size was significantly associated with higher rates of POHP. In patients with thyroid carcinoma, lymphadenectomy and the number of lymph nodes resected were associated with higher rates of POHP. Conclusion: The incidence of immediate postoperative hypoparathyroidism is within international standards. Standardized postoperative follow up is necessary, and strategies to improve POPH such as auto transplantation in locally identified highrisk subgroups should be considered.

Key words: Postthyroidectomy, Hypocalcaemia, Hypoparathyroidism

Ann Afr Surg. 2017; 14(2):76-81

DOI:http://dx.doi.org/10.4314/aas.v14i2.5

(C) 2017 Author. This work is licensed under the Creative Commons Attribution 4.0 International License.

hospital stays as well as an extra burden of medication and follow up for patients. Permanent hypoparathyroidism has further long term complications, such as nephrocalcinosis, renal dysfunction, basal ganglia calcifications and seizures (5, 6). Internationally, the reported incidence of POHP is variable ranging from 3 to $50 \%(4,7)$, depending on the definition used $(1,3)$. The use of multiple definitions forthis complication makes it very difficultto compare incidence across different studies.The British 
Association of Endocrine and Thyroid Surgeons (BEATS) most recent audit reported an incidence of transient postoperative hypocalcaemia of $24.9 \%(5)$. Previously identified predictors for POHP have included, amongst others, older age, female sex, surgery for Grave's disease, bilateral neck surgery, surgery for thyroid malignancy and size of thyroid gland (8). However, predictors for POHP also varied across different studies and in different locations, and even different studies in the same centers have produced conflicting information on predictors for POHP or hypocalcaemia (3). Limited data exists on the incidence of POHP and the factors which predict risk for this condition in South Africa. This study aims to calculate the incidence of POHP in a South African tertiary high volume setting, as well as identify the risk factors relevant to our population.

\section{Methods}

All patients who underwent a total thyroidectomy or completion thyroid lobectomy from January 2010 to December 2015 at the Groote Schuur Surgical Breast and Endocrine Oncology Unit were included in this study. Data was reviewed retrospectively from a prospectively maintained database. All operations were done by a standard trans-cervical open approach, and used a harmonic dissector to facilitate hemostasis. This is an academic training unit, with primary operating surgeons of varying experience, from senior subspecialist to trainee surgeon, supervised during surgery. The data reviewed included post-operative parathyroid hormone level, age, gender, weight and dimensions of thyroid specimen resected, extent of surgery and if lymphadenectomy was done, type of lymphadenectomy, presence of extracapsular extension of carcinoma, number of lymph nodes present in the specimen, presence and number of parathyroids in the specimen and final histological diagnosis of thyroid pathology. Central lymphadenectomy refers to resection of the nodes in level 6 of the neck, and lateral lymphadenectomy implies resection of nodes from level 2a, 3 and 4 of the neck. Following a total or completion thyroidectomy, the unit protocol requires serum $\mathrm{PTH}$ to be drawn 6-12 hours postoperatively. Patients were defined as having POHP if the postoperative serum iPTH level was less than 1.6pmol/l (lower limit of normal in the assay used in our laboratory). The records of these patients were then assessed for surgical and patient risk factors which have been shown in international cohorts to be associated with POHP. The pathology reports were analyzed for information about the size and weight of the resected thyroid specimens, presence of parathyroids and number of lymph nodes resected. Volume was calculated by multiplying anterior-posterior, cranio-caudal and transverse measurements of the resected specimens. All histological subtypes of carcinoma were grouped under 'malignant', while all other diagnoses were grouped into the 'benign' group. Descriptive statistics were performed on the patients, followed by Wilcoxon signed rank tests to explore the relationship between volume and weight and the outcome of postoperative hypoparathyroidism. Fishers' exact, Pearson chisquared and 2 sample proportion tests were used to explore relationships between the outcome and gender, cancer and lymphadenectomy while taking into account the numbers in the sample. Significance level was set at alpha $\leq 0.05$. Pearson's correlation was used to investigate the linear relationship between weight and volume of the thyroid tissue removed.

\section{Results}

The records of 203 patients were captured for this study. Papillary thyroid cancer $(31 \%)$ and multi-nodular goiter (29\%) were the most common histological diagnoses (Table1). The patients had a mean age of 47.6 years and $85 \%$ were female. The distribution of histological results for malignant and benign patients is shown in table 1. Papillary thyroid cancer was the most common malignant histological diagnosis, while multinodular goiter (MNG) was the most common benign diagnosis. Only one thyroid cyst was seen along with one case of Hurthle cell adenoma. Lymphoma and papillary microcarcinoma were rare cancers in this series.

Overall 59 patients (29.1\%) developed immediate postoperative hypoparathyroidism. Their characteristics are described in Table 2.

Gender did not significantly affect the outcome in this group. Age was not significantly associated with increased risk of POHP, and whether or not the underlying diagnosis was benign or malignant did not 
play a role either. The numbers of thyroidectomies for Grave's disease were too small to assess.

Table 1: Distribution of Histological Results

\begin{tabular}{|l|c|}
\hline Total number of patients & 203 \\
\hline Histology results & \\
\hline Total malignancies & $\mathbf{9 3 ( 4 5 . 8 \% )}$ \\
\hline Papillary Thyroid Cancer & $63(31.03 \%)$ \\
\hline Follicular Thyroid Cancer & $15(7.39 \%)$ \\
\hline Medullary Thyroid Cancer & $7(3.45 \%)$ \\
\hline Hurthle Cell Carcinoma & $5(2.46 \%)$ \\
\hline Lymphoma & $1(0.49 \%)$ \\
\hline Papillary microcarcinoma & $1(0.49 \%)$ \\
\hline Poorly Differentiated Carcinoma & $1(0.49 \%)$ \\
\hline Total Benign Diagnoses & $\mathbf{1 1 0}(\mathbf{5 4 . 2 \% )}$ \\
\hline MultinodularGoitre & $59(29.06 \%)$ \\
\hline Hashimoto's thyroiditis & $12(5.91 \%)$ \\
\hline Normal completion thyroidectomies & $12(5.91 \%)$ \\
\hline Graves' Disease & $7(3.45 \%)$ \\
\hline Thyroiditis & $7(3.45 \%)$ \\
\hline Follicular adenoma & $5(2.46 \%)$ \\
\hline Nodular hyperplasia & $3(1.48 \%)$ \\
\hline Toxic Multinodular Goitre & $3(1.48 \%)$ \\
\hline Hurthle cell adenoma & $1(0.49 \%)$ \\
\hline Thyroid cyst & $1(0.49 \%)$ \\
\hline
\end{tabular}

Table 2: Relationship between Gender, Age, Parathyroid in Specimen and Cancer Status of Patients

\begin{tabular}{|c|c|c|c|c|}
\hline \multirow[t]{2}{*}{ Characteristics } & \multicolumn{2}{|c|}{ Post-operative PTH } & \multirow[b]{2}{*}{$\begin{array}{l}\text { Total } \\
N= \\
203\end{array}$} & \multirow[b]{2}{*}{$\begin{array}{l}\mathrm{P} \\
\text { value }\end{array}$} \\
\hline & $\begin{array}{l}\text { normal } \\
\text { post op } \\
\text { iPTH } \\
144 \\
(70.9 \%)\end{array}$ & $\begin{array}{l}\text { low post } \\
\text { op iPTH } \\
59 \\
(29.1 \%)\end{array}$ & & \\
\hline \multicolumn{5}{|l|}{ Gender } \\
\hline Male & $24(17 \%)$ & $10(17 \%)$ & 34 & \\
\hline Female & $\begin{array}{c}120 \\
(83 \%)\end{array}$ & $49(84 \%)$ & 169 & 0.961 \\
\hline Age mean (SD) & $47.8(14)$ & $\begin{array}{c}46.9 \\
(13.8) \\
\end{array}$ & 202 & 0.6773 \\
\hline $\begin{array}{l}\text { No parathyroid } \\
\text { tissue present } \\
\text { in specimen }\end{array}$ & 129 & 47 & & 0.059 \\
\hline \multicolumn{5}{|l|}{ Cancer } \\
\hline No & $82(57 \%)$ & $28(48 \%)$ & 110 & 0.3885 \\
\hline Yes & $62(43 \%)$ & $31(53 \%)$ & 93 & 0.3874 \\
\hline
\end{tabular}

iPTH-intact Parathyroid Hormone; SD-Standard Deviation

Parathyroids were only inadvertently removed in 27 cases $(13 \%)$. The presence of parathyroids in the specimen was not significantly associated with low post-operative iPTH. The chance of parathyroids being present in the specimen was also not significantly increased if the operation was done for a malignancy.

There was a significant relationship between volume and POHP in patients who had surgery for benign thyroid disease, with a significant increase of POHP in those with a thyroid volume greater than $200 \mathrm{~cm} 3$. There was no volume correlation in patients who underwent surgery for malignancy. Although initial analysis indicated no significant difference between malignant and benign patients' risk of developing POHP, it was postulated that the disease processes should be considered separately to the additional surgeries for malignancies, and may have different risk factors. They were analyzed separately

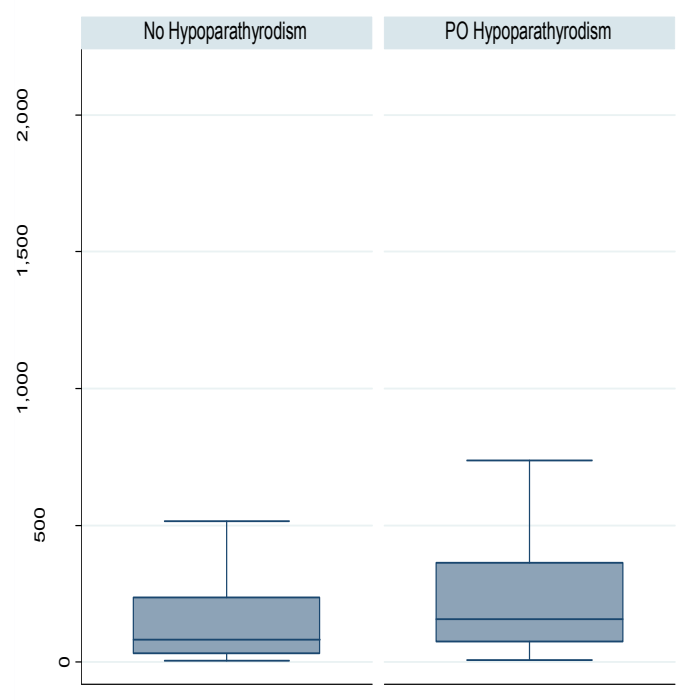

Figure 1 A box plot of the volume of thyroid specimen resected in patients with and without Post-operative hyperparathyroidism

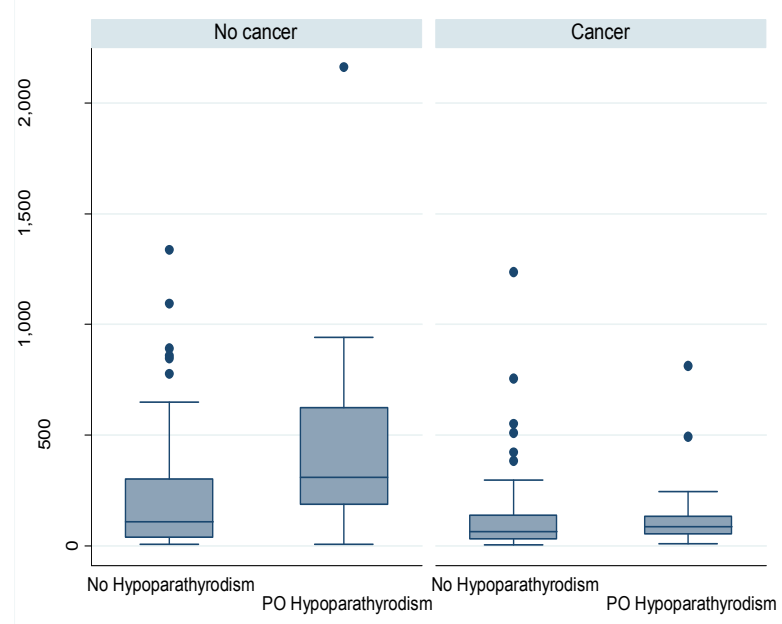

Figure 2 Box plots of the weight of thyroid specimen in patients with and without cancer by postoperative hypoparathyroidism status 
Table 3: Distribution of Study Characteristics According to Post-operative Hypoparathyroidism Status

\begin{tabular}{|c|c|c|c|c|c|c|c|c|}
\hline \multirow[t]{4}{*}{ Variable } & \multicolumn{8}{|c|}{ Cancer status } \\
\hline & \multicolumn{4}{|l|}{ Benign patients } & \multicolumn{4}{|c|}{ Malignancy patients } \\
\hline & \multicolumn{4}{|c|}{ Post-operative hypothyroidism } & \multicolumn{4}{|c|}{ Post-operative hypothyroidism } \\
\hline & No $n(82)$ & Yes n(28) & $\begin{array}{l}\text { Total } \\
\mathbf{n}=110\end{array}$ & $\begin{array}{l}P \\
\text { values }\end{array}$ & No $n(62)$ & Yes n(31) & $\begin{array}{l}\text { Total } \\
\mathrm{n}=93\end{array}$ & $P$ values \\
\hline \multicolumn{9}{|l|}{ Gender } \\
\hline Male & $10(12.20 \%)$ & $1(3.57 \%)$ & 11 & & $14(22.60 \%)$ & $9(29.03 \%)$ & 23 & \\
\hline Female & $72(87.80 \%)$ & $27(96.43 \%)$ & 99 & 0.284 & $48(77.40 \%)$ & $22(70.97 \%)$ & 70 & 0.497 \\
\hline Age median (IQR) & $48.5(37-57)$ & $51.5(38.5-57)$ & 110 & 0.8088 & $52(38-61)$ & $45(37-58)$ & 92 & 0.4933 \\
\hline $\begin{array}{l}\text { Tissue Weight (gr) } \\
\text { median (IQR) }\end{array}$ & $62(24-169)$ & $150(134-296)$ & 25 & 0.0030 & $\begin{array}{l}27.5(11.7- \\
72)\end{array}$ & $\begin{array}{l}38.5(24.1- \\
109.5)\end{array}$ & 20 & 0.1340 \\
\hline $\begin{array}{l}\text { Tissue Volume } \\
\left(\mathrm{cm}^{3}\right) \text { median } \\
(\mathrm{IQR})\end{array}$ & $109.9(37-303)$ & $309(188-624)$ & 105 & 0.0013 & $\begin{array}{l}64(28.8- \\
139.6)\end{array}$ & $\begin{array}{l}85.35(53.25- \\
133.15)\end{array}$ & 83 & 0.2598 \\
\hline $\begin{array}{l}\text { Nodes median } \\
\text { (IQR) }\end{array}$ & $0(0-0)$ & $0(0-0)$ & 89 & 0.2654 & $0(0-5)$ & $1(13-37)$ & 86 & 0.0060 \\
\hline \multicolumn{9}{|l|}{ Procedure } \\
\hline $\begin{array}{l}\text { Completion } \\
\text { lobectomy }\end{array}$ & $20(24.39 \%)$ & $4(14.28 \%)$ & 24 & 0.2634 & $15(24.19 \%)$ & $4(12.90 \%)$ & 19 & \\
\hline $\begin{array}{l}\text { Total } \\
\text { Thyroidectomy }\end{array}$ & $59(71.95 \%)$ & $20(71.42 \%)$ & 79 & 0.9571 & $47(75.80 \%)$ & $27(87.10 \%)$ & 74 & 0.278 \\
\hline Missing & $3(3.67)$ & $4(14.28 \%)$ & 7 & 0.0472 & - & - & - & \\
\hline \multicolumn{9}{|l|}{ Lymphadenectomy } \\
\hline No & $81(98.80 \%)$ & $24(85.70 \%)$ & 105 & & $35(56.45 \%)$ & $7(22.58 \%)$ & 42 & \\
\hline Yes & $1(1.20 \%)$ & $4(14.30 \%)$ & 5 & 0.015 & $27(43.55 \%)$ & $24(77.42 \%)$ & 51 & 0.002 \\
\hline \multicolumn{9}{|l|}{$\begin{array}{l}\text { Types of } \\
\text { lymphadenectomy }\end{array}$} \\
\hline Central and lateral & $1(1.20 \%)$ & - & 1 & - & $12(19.40 \%)$ & $16(51.61 \%)$ & 28 & 0.0014 \\
\hline Central & - & $4(14.30 \%)$ & 4 & - & $15(24.20 \%)$ & $8(25.80 \%)$ & 23 & 0.8661 \\
\hline \multicolumn{9}{|l|}{ Extra-capsular } \\
\hline No & - & - & - & - & $33(53.23 \%)$ & $16(51.61 \%)$ & 49 & 0.8827 \\
\hline Yes & - & - & - & - & $10(16.13 \%)$ & $9(29.03 \%)$ & 19 & 0.1458 \\
\hline
\end{tabular}

Table 3 shows the distribution of study characteristics stratified by POHP and cancer status. 110 had benign diagnoses and 93 had malignant histology. For benign patients, weight and volumewere significantly associated.

The median weight of the resected thyroid specimens of $62 \mathrm{gr}$ was lower for patients with no POHP compared to those with positive operative hypothyroidism (150gr) $(p=0.00030)$. The median volume of resected specimens was statistically different $(\mathrm{p}=0.0012)$ between patients without POHP (109.9 (IQR 37-303) and those who were found to be having POHP 309 (IQR 188-624). For patients with malignant histology, the number of lymph nodes resected, lymphadenectomy, and extent of lymphadenectomy showed statistically significant differences with regard to POHP status. The relationship between lymphadenectomy and POHP was statistically significant $(p=0.002) .16$ patients who underwent both central and lateral lymphadenectomydeveloped POHP (51.61\%), a statistically significant difference $(p=0.0014)$.A diagnosis of malignancy alone was not a predictor for postoperative hypoparathyroidism 0.3874), butmalignancypatients undergoing lymphadenectomy had a significantly higher proportion of postoperative hypoparathyroidism $(p=0.0179)$.

This suggests that the risk factor is in fact undergoing a lymph node dissection, rather than the malignancy itself. The patients with a greater number of nodes removed were also more likely to develop POHP ( $p=$ 0.0060).

\section{Discussion}

It is commonly accepted that thyroid surgery is complex and should be performed in a high volume unit (9).Even within a high volume center with experienced surgeons, POHP is common. In order to improve patient outcomes, it is necessary to measure the incidence of this problem and then identify the risk factors to evaluate whether practice can be modified to decreasethe complication rate. In our tertiary referral unit, $29 \%$ of patients undergoing total or completion thyroidectomy in the past five years were diagnosed 
with postoperative hypoparathyroidism. We defined postoperative hypoparathyroidism as serum iPTH of less than 1.6pmol/1, measured within 6-12 hourspostoperatively. Other units have used differentdefinitions, making comparison of incidences challenging (3). Some units measure only postoperative calcium, and plot a trend of serum calcium to predict whether or not the patient will develop symptomatic hypocalcaemia. Some units measure both iPTH and serum calcium. A 2008 review showed that an early low postoperative PTH correlates closely with postoperative hypocalcaemia (10), allowing us to perform a single blood test to determine if postoperative hypoparathyroidism is present. Our incidence of postoperative hypoparathyroidism (29\%) falls well within quoted ranges (11) and is close to the BAETS quoted incidence of postoperative hypocalcaemia. Despite using different markers, this provides the same information. This result indicates that we are working to international standards (5)

In benign disease, only the weight and volume (closely related) of the specimen was a significant risk factor for development of postoperative hypoparathyroidism. It is easy to understand how a larger volume of tissue in a limited space makes an operation more technically challenging. Parathyroids are small glands, difficult to identify, with an average weight of around only $60 \mathrm{mg}(12)$. The risk factors we have identified may also have to do with the distribution of disease we operate on in our unit. Autoimmune thyroid disease was identified as a risk factor for postoperative hypoparathyroidism by the American College of Endocrinology (8). However, only $3.45 \%$ of our thyroidectomies done over five years were for Grave's Disease, meaning that the numbers are too small to allow comment on the significance of this as a risk factor.

In patients with thyroid cancer, lymph node dissection was a risk factor for postoperative hypoparathyroidism. The need for a neck dissection reflects a more advanced stage cancer. However, the presence of extracapsular extension or excised parathyroids on histology is not significant for POHP. This implies that it is the inadvertent removal of parathyroid tissue during the lymphadenectomy specimen that is the problem rather than deliberate removal of parathyroid glands invaded by the malignant thyroid tissue. This indicates that if better identification of parathyroid tissue intraoperatively were to occur, parathyroid tissue could be preserved in-situ or auto-transplanted, and the incidence of POHP decreased. There are multiple devices either commercially available or under development to address this issue, but they are not yet feasible for widespread use.

The identification of key risk groups in our practice: patients with malignancy, who undergo neck dissection and those who have a large multinodular goiter, allows us to target these groups for specific parathyroid preserving strategies. Alternate strategies of parathyroid tissue identification and preservation should be considered and investigated. Newer technologies such as immunofluorescence are being explored, but are far from widespread use (13). In high risk patients a low threshold for auto transplantation of ischemic parathyroids should be considered. The best techniques for auto transplantation should be standardized and widely taught. The data was not suitable for a regression analysis and this may have limited our ability to identify risk factors. There were few male patients in this study and this may have limited our ability to detect a difference by sex. The investigation of an effect of age may have been limited by the study containing very few patients at the extremes of age. The majority were middle aged. A major shortcoming of this study was the inability to document the incidence of temporary versus permanent hypoparathyroidism in our patients. During the study period, follow up of these patients was not standardized and not all done through the surgical service. Many patients did not have regular repeat iPTH levels and where these were done, we were not always able to retrospectively access the result. Also, the documentation of length of hospital stay and long term adverse outcomes was not available. Improvements in these aspects of the post-operative care pathway as well as more consistent measurement of Vitamin D levels which has been noted to be a cause of postoperative hypocalcaemia(8), are some of the interventions that will flow from the conclusion of this research.

\section{Conclusion}

The incidence of postoperative hypoparathyroidism after total thyroidectomy in our unit is $29 \%$. The main 
risk factors for POHP in our context are large size of the thyroid gland and lymphadenectomy (and specifically the extent of the lymphadenectomy). While the incidence of this complication is within international standards, the adverse clinical impact may be greater in our setting as many patients are not able to access adequate follow up including appropriate investigations and treatment of POHP and its complications. Every effort should be made to minimize the incidence of POHP.

\section{References}

1. Lorente-Poch L, Sancho JJ, Sitges-Serra A et al. Defining the Syndromes of Parathyroid Failure after Total Thyroidectomy. Gland Surg. 2015; 4(1):8290.

2. Grodski S, Serpell J. Evidence for the Role of Perioperative PTH Measurement after Total Thyroidectomy as a Predictor of Hypocalcemia. World J Surg. 2008; 32(7):1367-73.

3. Edafe O, Antakia R, Balasubramanian SP, et al. Systematic Review and Meta-analysis of Predictors of Post-thyroidectomy Hypocalcaemia. Br J Surg. 2014; 101(4):307-20.

4. Cavicchi O, Piccin O, Caliceti U, et al. Accuracy of PTH Assay and Corrected Calcium in Early Prediction of Hypoparathyroidism After Thyroid Surgery. Otolaryngol Head Neck Surg. 2008; 138(5):594-600.

5. Edafe O, Prasad P, Balasubramanian SP et al. Incidence and Predictors of Post-thyroidectomy Hypocalcaemia in a Tertiary Endocrine Surgical Unit. Ann R CollSurg Engl. 2014; 96(3):219-23.

6. Powers J, Joy K, Ruscio A, et al. Prevalence and Incidence of Hypoparathyroidism in the United States Using a Large Claims Database. J BMR 2013; 28(12):2570-6.

7. Bellantone R, Lombardi CP, De Crea C, et al. Is Routine Supplementation Therapy (calcium and vitamin D) Useful after Total Thyroidectomy? Surgery. 2002; 132(6):1109-13.

8. Stack BC, Bimston DN, Orloff LA, et al. American Association of Clinical Endocrinologists and American College of Endocrinology Disease State Clinical Review: Postoperative Hypoparathyroidism
- Definitions and Management. EndocrPract. 2015; 21(6):674-85.

9. Nouraei S, Virk J, Vaz F, et al. A National Analysis of Trends, Outcomes and Volume - Outcome Relationships in Thyroid Surgery. ClinOtolaryngol 2017; 42(2): 354-65

10. Grodski S, Farrell S. Early Postoperative PTH Levels as a Predictor of Hypocalcaemia and Facilitating Safe Early Discharge after Total Thyroidectomy. Asian J Surg. 2007; 30(3):178-82.

11. Cavicchi O, Piccin O, Ceroni AR, et al. Transient Hypoparathyroidism Following Thyroidectomy: A Prospective Study and Multivariate Analysis of 604 Consecutive Patients. Otolaryngol - Head Neck Surg. 2007; 137(4):654-8.

12. Yao K, Singer FR, Giuliano AE, et al. Weight of Normal Parathyroid Glands in Patients with Parathyroid Adenomas. J ClinEndocrinolMetab. 2004; 89(7):3208-13.

13. McWade MA, Sanders ME, Mahadevan-Jansen A, et al. Establishing the Clinical Utility of Auto fluorescence Spectroscopy for Parathyroid Detection. Surg. 2016; 159(1):193-202. 